\title{
A Multi-objective model for selection of projects to finance new enterprise SMEs in Colombia
}

\author{
J. R. Coronado-Hernández ${ }^{1}$, E.M. Pardo-Mora ${ }^{2}$, M. Valero-Herrero ${ }^{3}$ \\ ${ }^{1}$ Universidad Tecnológica de Bolivar (COLOMBIA), ${ }^{2}$ Universidad de la Salle (COLOMBLA), \\ ${ }^{3}$ Universitat Politècnica de València (SPAIN) \\ jcoronado@unitecnologica.edu.co; empardo@unisalle.edu.co;mavaher@upv.es
}

Received June 2010

Accepted September 2011

\section{Abstract:}

Purpose: This paper presents a multi-objective programming model for selection of Projects for Financing New Enterprise SMEs in Colombia with objectivity and transparency in every call.

Approach: The model has four social objectives, subject to constraint budget and to the requirements of every summons. The resolution procedure for the model is based on principles of goal programming.

Findings: Selection projects subject to the impact within the country.

Research limitations: The selection of the projects is restricted by a legal framework, the terms of reference and the budget of the summons.

Practical implications: The projects must be viable according to the characteristics of every summons.

Originality/value: The suggested model offers an alternative for entities that need to evaluate projects of co-financing for the managerial development of the SMEs with more objectivity and transparency in the assignment of resources. 
Keywords: project selection, calls, SMEs, generation of employment in Colombia, multiobjective model

\section{Introduction}

The SMEs play an important role for the economic growth of Colombia. They generate $63 \%$ of the employment, $45 \%$ of the manufacturing production, $40 \%$ of salaries and $37 \%$ of the added value as shown in (Arcila, 2009), demonstrating its impact on the generation of wealth, growth and economic development. The defining policies and establishment of a legal agreement and of specific support for the support of the SMEs are relatively new in Colombia. They are the result of the 590 law of 2000 (Congreso de la República de Colombia, 2000) and extended by the law 905 of 2004 (Congreso de la República de Colombia, 2004) with the goal of promoting the integral development of SMEs. It is considered their capability to generate employment, regional development, the integration between economic sections, the productive advantage of small capitals taking into consideration the business capacity of the Colombians, and stimulating the promotion and formation of highly competitive markets through the fostering of the permanent creation and function of the greatest number of micro, small and medium enterprises.

With the 1014 law of 2006 (Congreso de la República de Colombia, 2006) a competitive spirit is promoted in all the educative classes in the country, in which they are inclined towards and work together on the principles and values of the legal and institutional framework, that motivate the start and creation of businesses. With the 905 law of 2004 (Congreso de la República de Colombia, 2004) the Colombian Fund for Modernization and Technological Development of Micro, Small, and Medium Enterprises (FOMIPYME) was created, ascribed to the Ministry of Commerce, Industry and Tourism (MCIT) with the goal of being an instrument of support in the politics of business development of the country. The fundamental objectives are: i) finance programs, projects and activities for the business and technological development of SMEs; ii) apply non-financial instruments directed to the fostering and promotion of SMEs, through nonrefundable co-funding of programs, projects and activities; iii) support the policies of business development, productive, commercial technological and of innovation that advances the MCIT. FOMIPYME opens summons (calls) every year to cofinance programs and projects of micro, small and medium enterprises; misplaced and productive mini-systems. In this work, a multi-objective mathematical model is 
proposed to select projects to co-finance in the calls of FOMIPYME framed in the law 905 of 2004 as assistance for making strong, objective and clear decisions in the selection of projects to co-finance.

Multi-criteria are used to locate an efficient solution of all the objectives although it is not an optimal solution with respect to each one of them, but rather one considering the objectives in conjunction. Benjamin in (1985) proposed a goalprogramming model for public-sector project selection in Trinidad and Tobago in which the goals are stipulated by the program manager. Barbarosoglu and Pinhas (1995) project selection tool for the Istanbul Water and Sewerage Administration used AHP and mixed integer programming (MIP) to include social, political and economic criteria. (Chan, 2004) has shown limited adoption by government units of those project selection and capital budgeting models proposed in the operations research literature. (Chan, DiSalvo \& Garrambone, 2005) used a goal-seeking methodology within a capital budgeting framework in considering technology modernization by the US Army. (Ghasemzadeh, Archer \& Iyogun, 1999) and (Medaglia, Hueth, Mendieta \& Sefair, 2008) integrate the different objectives into a single function by assigning different weighting scores to each objective according to their importance to the decision-maker. (Carazo et al., 2010) proposes a nonlinear combinatorial multi-objective model which simultaneously combines the selection and scheduling of project portfolios under general conditions making it applicable to public and private settings.

The solution procedure used in this article is based on principles of goal programming which try to explore the various trade-offs within the objectives. In section two, is presented a step in the summons to select project in FOMIPYME. In section three, a proposal is presented for the selection of projects based on multiobjective programming. In section four, a solution strategy based on goal programming is presented, which requires solving a set of fully mixed programming models that seek a compromise between the objectives to satisfy the criteria of the convocation and the development policies. Finally, we present a conclusion on the paper and give possible future research venues.

\section{Steps in the calls to select projects}

The process begins when $\mathrm{MCIT}$ opens calls in the resource assignment to FOMIPYME based on certain previous terms of reference approved by the administrative counsellor. They make a public convocation in which a proponent structure is proposed, presented and registered. Then, the proposals presented go 
into an evaluation process done by the Development Projects Financing Fund (FONADE) and the Corporation for the Development of Micro-businesses (CORPOMIXTA).The first of them evaluates the SMES' proposals and the second one evaluates the micro-businesses' proposals. The criteria analyzed in the process of evaluation, once the proposal fulfils the requirements of eligibility (minimum requirements established in the reference terms) to determine if it is viable are: the capacity of the proponent entity and the team executor, structure and coherence of the proposal and sector and regional pertinence. Based on the old criteria, the proposals obtained a ranking punctuation that should be superior to a minimum establishment previously to be considered viable. As noted, this ranking system is associated with expert criteria, which based on the documents, qualify and determine a ranking.

After determining which projects fulfil the minimum conditions with the ranking assigned, the following step is the approval of the proposals in which the evaluating identity holds up the proposal to the Technical Committee and the variables are presented to the Administrative Counsellor, who decides if it is approved or if the assigned proposal is not enough. In case that a sufficient proposal does not exist for the co-financing of the totality of the proposals, the selection criteria is the ranking obtained in the evaluation (it is in this point in which the multi-objective model proposal come in hand as selection criteria). The proposals recommended by the Technical Committee are taken to the FOMIPYME Administrative Counsel for approval. Then the eligible proposals pass to the signing of a co-financing contract between the proponent and FOMIPYME. Lastly, the project is initiated and the proponent contracts with an inventor that realizes the carrying out of project goals.

\section{Selection of the projects based on multi-objective programming}

In this section, a different alternative is presented, not based on the point ranking of the qualification obtained. We propose a multi-objective approximation considering criteria such as examples generated and/or obtained, the number of people benefited, coverage of the proposals and the points obtained, in such a way that a better possibility to carry out the main objectives in generating and maintaining the employment, benefitting the Colombian enterprises SMEs and cover all the zones of the country when the disposability of resources does not allow for the co-financing of all the variable proposals and at the same time select the best projects. 
The groups of projects that are eligible in this model are those that meet all of the minimum requirements. The combination of locations is related with the department or city (for the case of Bogotá, Cali and Medellin) which would cover the proposal. The type of beneficiary are the SMEs or Micro-businesses cannot be associated with illicit behaviour, a situation that is verified though the "Clinton List" and the Risk Management System for Money Laundering and the Financing of Terrorism (SARLAFT). The sector to which the people or beneficiary companies of the proposal belong can be a type of industry (IND), Artisans (ART), Services (SER), agricultural (AGR) o Mixed (MIX). The type of project is related with its own objective which can be: innovation (INN), business creation (CE), business improvement (MEJ), commercialization (COM) o Mini Productive Supply Chain (MINICA). These aspects have been included to consider that with accordance to the convocation some would have to be prioritized, value that is considered in the grading of each project of the candidate list depending on the policies of FOMIPYME.

\subsection{Mathematical Model}

In this section, different restrictions and objectives are used that in accordance with the objective of each convocation and whose structure permit the adaptation of change in policies, demonstrating that these types of problems can be modelled as a problem of optimization and can obtain a coherent, applicable, clear and strong response. The selection of the projects to co-finance is conditioned to the impact that the project has within the country in terms of the number of jobs generated and/or maintained covered by the project, moreover seeking a better number of beneficiary companies and selecting the best projects within the candidates list. The set of the model are:

$\begin{array}{ll}i \in I & \text { Set of Project } \\ d \in D & \text { Set of places } \\ t \in T & \text { Set of type of beneficiaries } \\ p \in P & \text { Set of bidder } \\ s \in S & \text { Set of sectors } \\ o \in O & \text { Set of project types }\end{array}$

Within the convocations of FOMIPYME, the MCIT assigns a budget with the goal of co-financing the projects taking into consideration that a certain minimum number business or beneficiaries of the approved projects must be met, a number of 
projects for micro businesses, a number of small businesses, a number of minimum and maximum projects to approve by departments or city capitals, a number of projects per section, such as the minimum number of businesses for approval to those displaced by violence. All the parameters associated with this model are presented below.

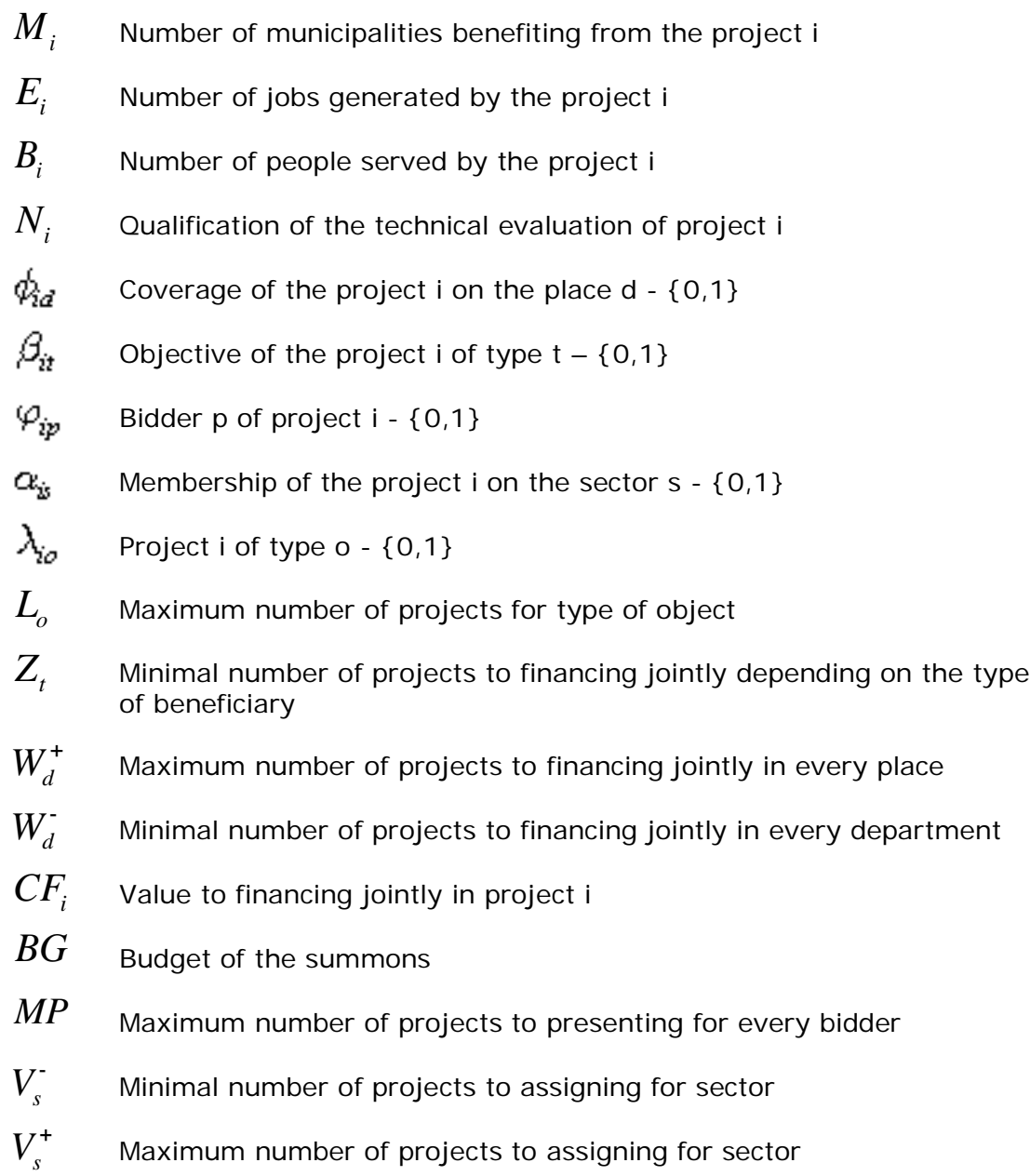

Among the variable declarations, each objective function ordered lexicographically has worth in accordance to the importance of the criteria partially to the direction of each summons, like a binary variable to determine the selected project and a variable to accumulate the budget surplus.

obj1 Number of persons benefited with the summons

obj2 Number of employments generated or supported by the summons

obj3 Number of municipalities covered by the summons

obj4 Qualification accumulated of the summons

$x_{i}= \begin{cases}1 & \text { If project is select. } \\ 0 & \text { Otherwise. }\end{cases}$ 
The expressions (1) to (4) represent the objectives of the model, where (1) total number of people benefited by the generation of businesses is maximized, in expression (2) the number of jobs generated is maximized; in (3) the benefiting population is maximized, and (4) the accumulated grade of the projects with the goal of selecting the best projects is maximized.

$$
\begin{aligned}
& \max \quad a b j 1=\sum_{i \in I} B_{i} x_{i} \\
& \max \quad a b j 2=\sum_{i \in I} E_{i} x_{i} \\
& \max \quad o b j 3=\sum_{i \in I} M_{i} x_{i} \\
& \max \quad o b j 4=\sum_{i \in I} N_{i} x_{i}
\end{aligned}
$$

The decision to optimize these objectives is restricted by a combination of constraints that limit the space of solutions and that characterize a legal agreement and presupposition of the summons. The expression (5) is the presupposed constraint, in which the sum total of the projects that are going to be co-financed should be less or equal to the presupposition of each summons, a variable $y$ is used to save the budget surplus. The expressions (6) and (7) refer to the minimum and maximum amount of projects that should be selected per location; when here is no maximum of projects to select, a Big M should be added within the vector in the specific position in the vector of the location parameter. The expression (8) shows the maximum number of projects that there should be per type of object, if a limitation exists and in this case when there is no maximum, a Big $M$ should be added within the vector on the right side. The expression (9) makes a reference to which depending on the type of beneficiary, a minimum number of projects should be selected. The constraint (10) shows what maximum a proponent can have MP projects; in principle the historical maximum of the past summons has been a maximum of two projects per bidder. The constraints (11) and (12) are those which are limit the minimum and maximum number of projects per sector. Finally, the obvious constraints in integrality and non-negativity are shown.

$$
\begin{array}{ll}
\sum_{i=I} C F_{i} x_{i} \leq D G-y & \\
\sum_{i=i} \phi_{i d} x_{i} \geq W_{i}^{-} & \forall d \in D
\end{array}
$$




$$
\begin{array}{ll}
\sum_{i=i} \phi_{i d} x_{i} \leq W_{d}^{+} & \forall d \in D \\
\sum_{i=I} \lambda_{i o} x_{i} \leq L_{0} & \forall o \in O \\
\sum_{i \in I} \beta_{i t} x_{i} \geq Z_{t} & \forall t \in T \\
\sum_{i \in I} \varphi_{i j} x_{i} \leq M P & \forall p \in P \\
\sum_{i \in I} \alpha_{i j} x_{i} \geq V_{s}^{-} & \forall s \in S \\
\sum_{i \in I} \alpha_{i j} x_{i} \leq V_{s}^{+} & \forall s \in S \\
x_{i} \in\{0,1\},, y \geq 0 & \forall i \in I
\end{array}
$$

\section{Solution Method and implementation}

For the resolution goal programming is used, which aims to optimize the objectives in an individual way, ordered lexicographically sequentially in order of importance and then place parameters around this objective and introduce it into a set of constraints $\Omega$ that limit the solution space represented in expression (5) to (13).

This way's solution is found for just one objective so as to reach goal for the other objectives not presents in the objective function to optimize. The model is executed in each state with the only one objective (Within each objective function different coefficients make differences within comparable elements). When a model is optimized with a goal, a new constraint is introduced in the model with a chosen level of slack (varying according to its nature) and the following model is resolved with another goal and with the introduction of new constraints (in the opportune case) until the ultimate goal is reached as observed in Figure 1. In this way the solution to the selection of the projects is obtained. The model is run again whereas there is a budget surplus and if this is less than the minimum value of the projects to co-finance of the projects that have not been selected. The model is turn again with the projects that were not selected in the previous running of the model and with the assumption of the value that obtains the primal worth in of the variable $y$. The model was implemented in the mathematical programming language AMPL over in Java using as an optimizer LPSolve 5.5. (Berkelaar, Eikland $\&$ Notebaert, 2005).

The general approach is shown in Figure 2. The process begins with the extraction from the data base of information on the parameters that feed the models whose inputs are in a XML (Extensible Markup Language) archive which contains all the 
information of the problem. In XML of the problem together with the mathematical model, each one of the objectives previously programmed generates an archive with the problem generated and is solved using LPSolve following the methodology shown in Figure 1, then writes the results in a document and the process continues if the budget surplus is more than the minimum value in the list of projects that have not been chosen.

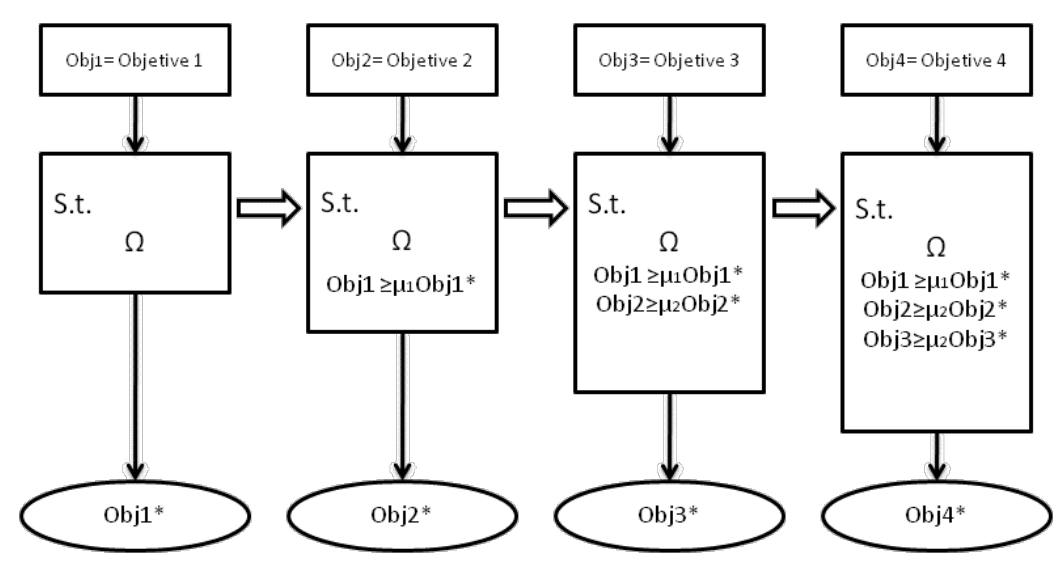

Figure 1. Visualization of the schema resolution

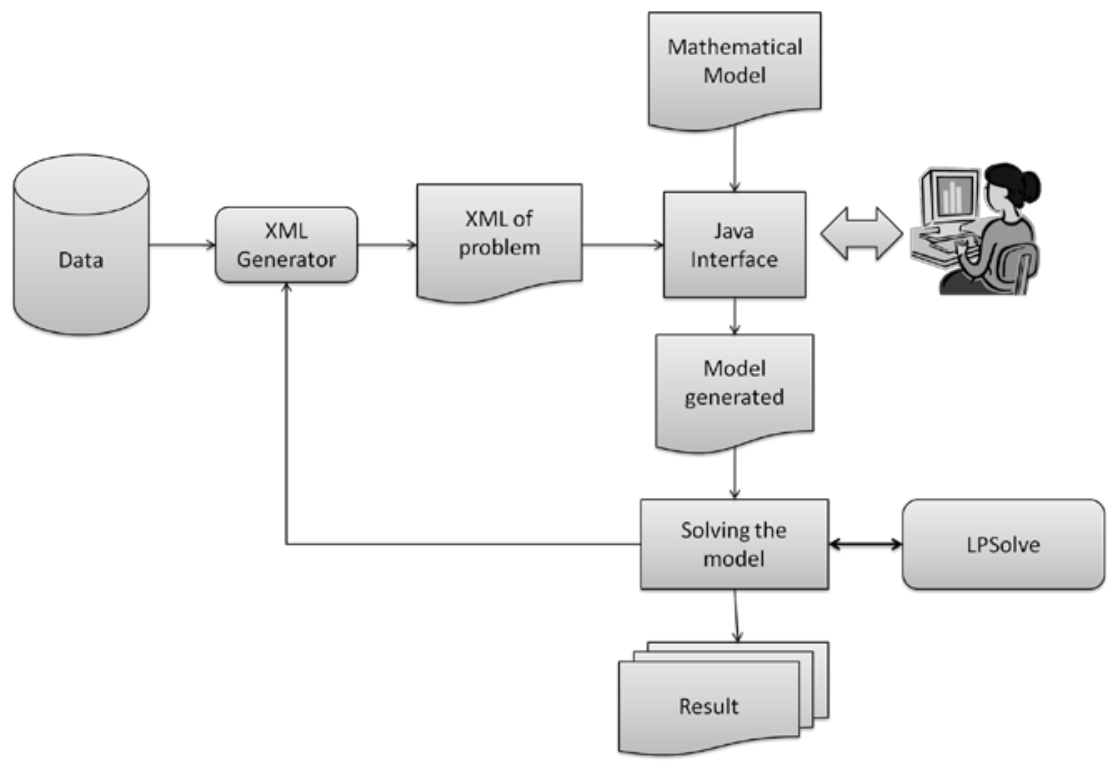

Figure 2. Schema of solution

\section{Conclusion}

In this work, a multi-objective mathematical model was proposed to select projects to co-finance in the convocations of FOMIPYME framed in the 905 law of 2004 as assistance for making strong, objective and clear decisions in the selection of projects to co-finance. The solution procedure used in this article is based on 
principles of goal programming which try to explore the various trade-offs within the objectives. Multi-criteria are used to locate an efficient solution of all the objectives although it is not an optimal solution with respect to each one of them, but rather one considering the objectives in conjunction. The procedure aims to optimize the objectives in an individual way, ordered lexicographically sequentially in order of importance and then place parameters around this objective and introduce it into a set of constraints that limit the solution space.

The selection of the projects to co-finance is conditioned to the impact that the project has within the country in terms of the number of jobs generated and/or maintained covered by the project, moreover seeking a better number of beneficiary companies and selecting the best projects within the candidates list. The model was implemented in the mathematical programming language AMPL over in Java using as an optimizer LPSolve. The use of this type of model to select the projects offers an alternative to those entities that are faced with a selection process similar to that of Fomipyme, in which they have clear objectives and preestablished goals so that more objectivity exists in the allocation of resources.

Inside work's future lines is found the incorporation of uncertainty in the parameters approaching the problem with Fuzzy Set and stochastic programming. On the other hand, the solution of the problem using the method e-constraint generates an efficient frontier and the metaheuristics application for the solution of multi-objective problems.

\section{Acknowledgments}

Thanks to FOMIPYME for their support in this project. This work has been supported by the Research Support Program of the Universitat Politècnica de València PAID01-10.

\section{References}

Arcila, J.A.G. (2009). Agencias MIPYMES, creación de empresas y desarrollo empresarial: una lectura sobre Colombia y Puerto Rico. Revista Panorama Económico, 16.

Barbarosoglu, G. \& Pinhas, D. (1995). Capital rationing in the public sector using the analytic hierarchy process. The Engineering Economist, 40, 315-341. http://dx.doi.org/10.1080/00137919508903158 
Benjamin, C.O. (1985). A linear goal-programming model for public-sector project selection. Journal of the Operational Research Society, 13-23.

Berkelaar, M., Eikland, K., \& Notebaert, P. (2005). Ipsolve: Open source (mixedinteger) linear programming system, version 5.5.0.15. [Computer software].

Carazo, A.F., Gomez, T., Molina, J., Hernandez-Diaz, A.G., Guerrero, F.M., \& Caballero, R. (2010). Solving a comprehensive model for multiobjective project portfolio selection. Computers \& Operations Research, 37, 630-639. http://dx.doi.org/10.1016/i.cor.2009.06.012

Chan, Y., DiSalvo, J.P., \& Garrambone, M.W. (2005). A goal-seeking approach to capital budgeting. Socio-Economic Planning Sciences, 39, 165-182. http://dx.doi.org/ 10.1016/i.seps.2004.04.002

Chan, Y.C.L. (2004). Use of capital budgeting techniques and an analytic approach to capital investment decisions in Canadian municipal governments. Public Budgeting \& Finance, 24, 40-58. http://dx.doi.org/10.1111/j.0275-1100.2004.02402003.x

Congreso de la República de Colombia. (2000). Ley 509 de 2000.

Congreso de la República de Colombia. (2004). Ley 905 de 2004.

Congreso de la República de Colombia. (2006). Ley 1014 de 2006.

Ghasemzadeh, F., Archer, N., \& Iyogun, P. (1999). A zero-one model for project portfolio selection and scheduling. Journal of the Operational Research Society, 50, 745-755. http://dx.doi.org/10.1057/palgrave.jors.2600767

Medaglia, A.L., Hueth, D., Mendieta, J.C., \& Sefair, J.A. (2008). A multiobjective model for the selection and timing of public enterprise projects. Socio-Economic Planning Sciences, 42, 31-45. http://dx.doi.org/10.1016/i.seps.2006.06.009

Journal of Industrial Engineering and Management, 2011 (www.jiem.org)

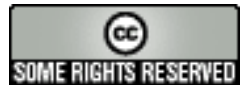

Article's contents are provided on a Attribution-Non Commercial 3.0 Creative commons license. Readers are allowed to copy, distribute and communicate article's contents, provided the author's and Journal of Industrial Engineering and Management's names are included. It must not be used for commercial purposes. To see the complete license contents, please visit http://creativecommons.org/licenses/by-nc/3.0/. 